\title{
The impact of an interactive workshop on the management of urinary catheterization on nurses
}

\author{
Insaf Altun and Ali Karakoç
}

\begin{abstract}
The aim of this study was to determine whether a structured workshop for nurses promoting best practice technique for management of indwelling urinary catheters results in an improvement in knowledge on the subject. A one-group pre-post test quasi-experimental design using a convenience sample was used. Nurses attended a workshop utilizing interactive lecture approaches, and based on best practice technique for the management of indwelling urinary catheters. Participants $(n=30,55 \%$ of those invited) completed a multiple choice question (MCQ) test, derived from topics to be covered in the workshop, prior to the intervention. The MCQ test was repeated after the workshop to assess retention and application of knowledge. There was a significant improvement in mean test scores after the workshop when compared with pre-workshop scores $($ mean $=16.9, \mathrm{SD}=1.1$ vs. mean $=8.5, \mathrm{SD}=1.7, p<0.001)$. It is concluded that interactive lecture workshops based on best practice techniques for the management of urinary catheterization help improve nurses' knowledge. Such educational initiatives also help to overcome deficiencies in initial nurse training where preparation for quality catheter care can be lacking. Within the limitations of a small-scale single-group study of a convenience sample, the information gained in this study will be valuable in helping to establish a baseline for further research. It may also help guide improvements in the implementation of policies for improved management of the care provided to people with an indwelling urinary catheter with the ultimate goal of enhancing safe and quality patient care.
\end{abstract}

Key words: Interactive lecture $\bullet$ Knowledge $\bullet$ Managing of urinary catheterization $\bullet$ Nurses $\bullet$ Test $\bullet$ Workshop

\section{BACKGROUND}

The proper management of urinary catheters is an often vital aspect of daily urological nursing care. Indwelling urinary catheters can be sited either urethrally or via the supra-pubic route and offer a closed sterile system to allow for full or controlled bladder drainage (Newman, 1998; Schumm and Lam, 2008; Sienkiewicz et al., 2008; Blodgett, 2009). Although the insertion of a urinary catheter typically follows a physician's request, it is increasingly the case that it is a nurse who often performs the catheterization

Authors: Dr i Altun, RN, PhD, Associate Professor, Department of Fundamentals in Nursing, Kocaeli University, Kocaeli, Turkey; A. Karakoç, RN, MSN, Ankara University, Cebeci Hospital, Ankara, Turkey

Address for correspondence: I Altun, Department of Fundamentals in Nursing, High School of Health, Kocaeli University, Umuttepe 41380, Kocaeli, Turkey

E-mail: ialtun@kocaeli.edu.tr and is responsible for its subsequent management (Marklew, 2004; Gray, 2008). Consequently, nurses have a responsibility to be aware of best practice issues to ensure that patients with indwelling urinary catheters receive the most appropriate care and information when caring for their indwelling urinary catheters (Cochran, 2007; Newman, 2007; Gotelli et al., 2008; Tsuchida et al., 2008; Parker et al., 2009a, 2009b). In addition, more positive patient outcomes are achieved when the risks of common catheterassociated complications, such as urethral and meatal tissue damage, infection, catheter encrustation and blockage are recognized and their effects minimized (Tenke et al., 2008; Blodgett, 2009; Gallegos, 2009; Nasiriani et al., 2009). An indwelling urinary catheter is associated with several complications that can significantly increase patient morbidity and mortality (Blodgett, 2009; Gallegos, 2009). One such problem is catheter-associated urinary tract infection (CAUTI), 
which is suggested to be a common and costly problem for hospitalized patients in particular (Cheung et al., 2008; Blodgett, 2009; Gallegos, 2009; Parker et al., 2009a, 2009b).

The proper management of an indwelling urinary catheter is one of the most common problems faced by patients and health professionals, both in hospital and primary health care settings, and a number of studies have reported on the problems relating to the management of an indwelling catheter (Tenke et al., 2008; Tsuchida et al., 2008; Gallegos, 2009; Parker et al., 2009a, 2009b). The role of the nurse has been suggested as being key to the assessment of appropriateness of continuing indwelling urinary catheter use and to the timely implementation of practices aimed at the identification and prevention of complications associated with this method of urinary drainage (Marklew, 2004; Gray, 2008).

While perhaps a truism, it is nonetheless essential that to ensure a standard of care that is high and consistent nurses must have appropriate levels of knowledge and skills as nursing interventions to help prevent complications are required during each phase of nursing care. However, many nurses remain unaware of the indications, complications and nursing practices that can prevent catheter-related adverse events (Williams et al., 2003; Drekonja et al., 2009; Willson et al., 2009). As stated, many nurses insert and manage catheters, yet some studies have shown that often nurses have limited scientific knowledge in the general area of catheters and their care (Williams et al., 2003; Drekonja et al., 2009; Willson et al., 2009). These studies emphasize the need for regular education for nurses and suggest that a multifaceted approach to tackle current deficiencies in knowledge should include the provision of evidencebased educational initiatives.

The aims of this study were to determine whether a structured interactive lecture workshop for nurses on the management of an indwelling urinary catheter would lead to an improvement in knowledge on the subject and be seen by participants as helpful in their provision of care.

\section{METHODOLOGY}

\section{Study design}

This pre-post test quasi-experimental descriptive study was conducted in a university hospital in Ankara, Turkey in November 2009.

\section{Participants}

Participants were 30 nurses working in a university hospital across the specialties of general surgery, orthopaedics, ENT, urology and spinal surgery. These represented a convenience sample of those nurses with a minimum of 4 years post-registration experience. Of those invited, 55\% $(n=30)$ agreed to participate. All participated in the workshop as part of an in-service training programme.

\section{Intervention}

Participants attended an interactive lecture-based workshop on indwelling urinary catheter management. Participants had accepted an invitation to the presentation prior to the training event. A pre-workshop multiple choice question (MCQ) test (Appendix A) was completed by participants to test their existing knowledge. The MCQs were derived from topics covered in the presentation. The MCQ items were obtained from sources used within this paper and from expert opinion.

Following the workshop, the MCQ test was repeated to assess both retention and application of knowledge delivered from the interactive lecture-based workshop. Participants were unaware that they would be tested with an MCQ prior to the workshop or that the MCQ would be repeated at the end of the workshop. Participants' consent was obtained regarding the use of their answers for the purpose of this study.

\section{Statistical analysis}

Statistical analysis of data was performed using the SPSS (SPSS for Windows, version 12.0). The Kolmogorov-Smirnov test was used to determine whether the data were normally distributed. The Student's paired $t$-test was used to determine whether the differences between the pre-workshop and postworkshop test results were significant. Differences were considered significant at $p<0.05$.

\section{RESULTS}

Thirty nurses participated in the workshop. There was a statistically significant improvement in test scores after the workshop when compared with pre-workshop scores (Table 1, Figure 1).

Table 1 Pre-workshop and post-workshop test scores

\begin{tabular}{lcc}
\hline & $\begin{array}{c}\text { Pre-workshop test } \\
(n=30)\end{array}$ & $\begin{array}{c}\text { Post-workshop test } \\
(n=30)\end{array}$ \\
\hline Mean (SD) & $8.5(1.7)$ & $16.9(1.1)$ \\
$95 \% \mathrm{Cl}$ & $7.9-9.2$ & $16.5-17.3$ \\
Kolmogorov-Smirnov test & 1.1 & 1.3 \\
Asymptotic significance & 0.17 & 0.05 \\
\hline
\end{tabular}

Maximum test score $=18$.

Paired sample Student's t-test (two-tailed) $p<0.001$. 


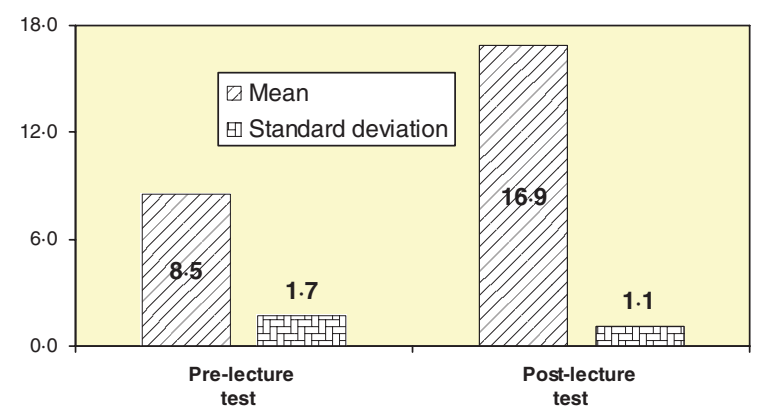

Figure 1 Pre-lecture and post-lecture test scores.

\section{DISCUSSION}

This study was undertaken to determine knowledge and practice for management of an indwelling catheter and to assess the effect of educational training given on this subject. Although nurses insert catheter and care for many patients with indwelling urinary catheters, little is known about nurses' attitudes, practice and knowledge regarding management of urinary catheters (Drekonja et al., 2009; Willson et al., 2009). Successful management of an indwelling urinary catheter requires that nurses have adequate knowledge relating to this subject (Drekonja et al., 2009; Willson et al., 2009). Completion of the workshop resulted in improved levels of knowledge.

Nurses in this study, having qualified for at least 4 years, would be expected to have at least a basic understanding of best practice for the management of an indwelling urinary catheter. But the mean preworkshop test score (mean $=8.5, S D=1.7$ ) was low. This suggests that, at the very least, for the participants there was a lack of proper preparation for this vital aspect of nursing care during initial nurse education and in the early years of their careers. A more effective form of teaching with explanation of the underlying concepts is required to improve knowledge and application of best practice technique for the management of an indwelling urinary catheter, as indicated by the significant improvement in postworkshop test scores (mean $=16 \cdot 9, \mathrm{SD}=1 \cdot 1$ ). This has also been indicated in other studies (Williams et al., 2003; Drekonja et al., 2009; Willson et al., 2009). However, while scores improved following the intervention described here, there is of course a huge difference between remembering something heard in a teaching session and actual 'knowing', retaining and utilizing what was learned in practice. Subsequently, it would be wrong to assume that all that would be required to improve both knowledge and care is didactic intervention alone. A sustained programme of initial education, observed practice and planned updating is necessary.
Overall, nurses acknowledged the importance and relevance of the subjects covered in the workshop and felt that it was worthwhile. However, limitations in this study must also be recognized. Among these were the limitations of convenience sampling and the small number of nurses in this study; 30 (approximately 55\%) of the nurses invited participated. This study tested immediate recall of knowledge and it remains to be seen whether the knowledge gained as a result of the event will be retained by the trainees and whether their application of best practice to management on an indwelling urinary catheter habits will be altered as a result. The authors believe that it would be useful to examine nurses' knowledge base and application of best practice for the management of an indwelling urinary catheter sometime after such an event to determine the need for continued and repeated training in this important subject. In addition, the improvement in the MCQ score could be at least partially attributed to an 'order effect'. It is possible that improvement in post-workshop scores could have happened without the structured workshop, simply because the nurses had the opportunity to think about the questions again and give a more considered answer. This might have been avoided if a randomized control trial design had been utilized, with participants subjected to the MCQ test randomized to control and intervention groups. Such approaches were not utilized in this small-scale preliminary study, and the findings must be considered in this light.

\section{CONCLUSION AND IMPLICATION FOR PRACTICE}

Nursing professionals must have sound (and where possible evidence-based) knowledge related to the management of an indwelling urinary catheter, complications caused by an indwelling urinary catheter and ways to prevent and manage these complications. We need not forget that nurses are health care professionals responsible for the application of best practice technique for the management of care in such circumstances.

Results of this study show that at the pre-programme phase, participant nurses' knowledge in relation to the management of an indwelling catheter was poor. This study has suggested that interactive workshops assessed by MCQs can improve nurses' knowledge on the topic. The findings might further suggest that greater emphasis needs to be placed on nurses' education of management of care practices associated with indwelling urinary catheters.

Providing nurses with information relating to management of an indwelling urinary catheter and supporting its retention and implementation are essential. 
These activities can promote adherence to best practice, self-assessment and self-reporting of the difficulties relating to the management of an indwelling urinary catheter. The results obtained in this study will be valuable as a baseline for further research and may aid improvement in the management of an indwelling urinary catheter, with the ultimate goal of enhancing high-quality patient-centred care.

The literature suggests that to practise safely nurses must have specific knowledge of the actions, benefits and risks associated with an indwelling urinary catheter. Finally, they need to be able to appropriately document assessment findings, decide when signs and symptoms indicate the likelihood of complications and implement appropriate actions if these complications arise. Thus, the main objectives of this study were to enhance nurses' knowledge and practice regarding the management of an indwelling urinary catheter and explore relationships between these variables and the education received by the nurses. This study examined nursing knowledge, the identification of actual or potential complications and clinical decisionmaking skills. The results of this study offer additional information to those tasked with initial and ongoing educational planning for learning associated with management of an indwelling urinary catheter. The range of clinical interventions undertaken by nurses globally, and not just within the specialty of urology, is at one and the same time ever increasing but lacking in global consistency. One thing does remain certain. No matter what the clinical activity, patients have the right to expect that the professional providing care has received appropriate education, is competent and that both their knowledge and competency are current and accurate. Ultimately, the patient is right to expect nothing less.

\section{WHAT IS KNOWN ABOUT THIS TOPIC}

- The proper management of urinary catheters is an often vital aspect of daily urological nursing care. But nurses have limited scientific knowledge in the general area of catheters and their care.

- Studies emphasize the need for regular education for nurses and suggest that a multifaceted approach to tackle current deficiencies in knowledge should include the provision of evidence-based educational initiatives.

\section{WHAT THIS PAPER ADDS}

- This study has suggested that interactive workshops assessed by MCQs can improve nurses' knowledge on the topic.

- The results of this study offer additional information to those tasked with initial and ongoing educational planning for learning associated with management of an indwelling urinary catheter.

\section{REFERENCES}

Blodgett TM. (2009). Reminder systems to reduce the duration of indwelling urinary catheters: a narrative review. Urologic Nursing; 29: 369-378; quiz 379.

Cheung K, Leung P, Wong YC, To OK, Yeung YF, Chan MW, Yip YL, Kwok CW. (2008). Water versus antiseptic periurethral cleansing before catheterization among home care patients: a randomized controlled trial. American Journal of Infection Control; 36: 375-380.

Cochran S. (2007). Care of the indwelling urinary catheter: is it evidence based? Journal of Wound, Ostomy $\mathcal{E}$ Continence Nursing; 34: 282-288.

Drekonja DM, Kuskowski MA, Johnson JR. (2009). Internet survey of Foley catheter practices and knowledge among Minnesota nurses. American Journal of Infection Control; 38: 31-37.

Gallegos SE. (2009). Healthcare-acquired catheter-associated urinary tract infections in a community hospital setting: how do we keep patients safe and keep reimbursement? American Journal of Infection Control; 37: E44 Poster Abstracts. Presentation number: 5-44.

Gray ML. (2008). Securing the indwelling catheter. American Journal of Nursing; 108: 44-50; quiz 50.

Gotelli JM, Merryman P, Carr C, McElveen L, Epperson C, Bynum D. (2008). A quality improvement project to reduce the complications associated with indwelling urinary catheters. Urologic Nursing; 28: 465-467, 473.

Marklew A. (2004). Urinary catheter care in the intensive care unit. Nursing in Critical Care; 9: 21-27.

Nasiriani K, Kalani Z, Farnia F, Motavasslian M, Nasiriani F, Engberg S. (2009). Comparison of the effect of water vs. povidone-iodine solution for periurethral cleaning in women requiring an indwelling catheter prior to gynecologic surgery. Urologic Nursing; 29: 118-121, 131.

Newman DK. (2007). The indwelling urinary catheter principles for best practice. Journal of WOCN; 34: 655-661.

Newman DK. (1998). Managing indwelling urethral catheters. Ostomy Wound Management; 44: 26-28, 30, 32, passim.

Parker D, Callan L, Harwood J, Thompson D, Webb ML, Wilde M, Willson M. (2009a). Clinical Practice Continence Subcommittee Catheter-associated urinary tract infections: fact sheet. Journal of Wound, Ostomy E Continence Nursing; 36: 156-159.

Parker D, Callan L, Harwood J, Thompson DL, Wilde M, Gray M. (2009b). Nursing interventions to reduce the risk of catheterassociated urinary tract infection. Part 1: catheter selection. Journal of Wound, Ostomy E Continence Nursing; 36: 23-34.

Schumm K, Lam TB. (2008). Types of urethral catheters for management of short-term voiding problems in hospitalized adults: a short version Cochrane review. Neurourology and Urodynamics; 27: 738-746. 
Sienkiewicz J, Wilkinson G, Emr KD. (2008). The quest for best practice in caring for the home care patient with an indwelling urinary catheter: the New Jersey experience. Home Healthcare Nurse; 26: 121-8; quiz 129-130.

Tenke P, Kovacs B, Bjerklund Johansen TE, Matsumoto T, Tambyah PA, Naber KG. (2008). European and Asian guidelines on management and prevention of catheterassociated urinary tract infections. International Journal of Antimicrobial Agents; 31: S68-S78.

Tsuchida T, Makimoto K, Ohsako S, Fujino M, Kaneda M, Miyazaki T, Fujiwara F, Sugimoto T. (2008). Relationship between catheter care and catheter-associated urinary tract infection at Japanese general hospitals: a prospective observational study. International Journal of Nursing Studies; 45 : 352-361.

Williams A, Taylor K, Bates C, Tincello DG, Richmond DH. (2003). Knowledge of female bladder care among medical staff, nurses and midwives: results of a questionnaire survey. BJU International; 91: 208-210.

Willson M, Wilde M, Webb ML, Thompson D, Parker D, Harwood J, Callan L, Gray M. (2009). Nursing interventions to reduce the risk of catheter-associated urinary tract infection: part 2: staff education, monitoring, and care techniques. Journal of Wound, Ostomy \& Continence Nursing; 36:137-154. 


\section{APPENDIX A}

For each given question select the single best answer from the choices provided ( $A-E)$.

1. What is the capacity of a normal bladder?
A $200 \mathrm{~mL}$
B $300 \mathrm{~mL}$
C $500 \mathrm{~mL}$
D $800 \mathrm{~mL}$
E $900 \mathrm{~mL}$

2. Which of the following statements is incorrect?
A The female urethra is approximately $7 \mathrm{~cm}$ long.
B The male urethra is approximately $18-20 \mathrm{~cm}$ long.
C Urethral lubrication is considered particularly important when catheterizing a male.
D Inadequate lubrication has been associated with urethral laceration and pain during insertion.
E Because of the extended length of the male urethra, $10 \mathrm{~mL}$ of lubrication is usually recommended.

3. Which of the following statements about aims of catheter care is true?
A To promote patient dignity and comfort
$B$ To recognize and minimize the risks of common catheter-associated complications, such as tissue damage, infection, catheter encrustation and blockage
C To assist patients in reaching their full potential in terms of self-care and independence
D To provide cost-effective service
E All of the above

4. Strategies to prevent CAUTIs have focused on:
A Catheter materials
B Drainage systems
C Insertion techniques
D Use of anti-infective agents
E All of the above

5. What size balloon should you use to keep a Foley Catheter in place?
A $5 \mathrm{~mL}$
B $10 \mathrm{~mL}$
C $20 \mathrm{~mL}$
D $30 \mathrm{~mL}$
E $40 \mathrm{~mL}$

6. Indications for use of an indwelling urinary catheter are:
A Urinary tract obstruction
B Nurse's concern about patient's discomfort
C Urinary retention
D Patient to undergo prolonged ( $>2 \mathrm{~h}$ ) procedure
E Recently underwent surgical/invasive procedure

7. Inappropriate indications for use of an indwelling urinary catheter are:
A Incontinence without any of the appropriate indications
B Stage III or IV skin ulcers
C Diuresis
D Frequent, but non-essential, determination of urinary output
E Diarrhoea, without any of the appropriate indications 
8. Indications for continued use of indwelling urinary catheter are:
A Aggressive treatment with diuretics or fluids
B Accurate monitoring of intake and output
C Patient's preference
D Relief of urinary retention not managed with intermittent catheterization
E Management of urinary incontinence with the terminally ill

9. Which of the following indications for short-term catheterization is incorrect?
A Urinary drainage following urologic, gynecologic, neurologic or abdominopelvic surgery
B Monitoring urine output in acute or critically ill patients
C Monitoring core body temperature
D Palliative care settings where routine toileting is compromised by pain or immobility
E Urinary drainage in patients with urinary retention

10. Which of the following indications for long-term indwelling catheterization is incorrect?
A Urinary retention associated with bladder outlet obstruction that cannot be managed by other methods
B Urinary incontinence coexisting with urinary retention that cannot be managed by other methods
C Delayed healing of a high-stage pressure ulcer owing to urinary incontinence
D Monitoring urine output in acute or critically ill patients
E Palliative care settings where routine toileting is compromised by pain or immobility

11. Adverse consequences of catheter-associated urinary tract infection include:
A Local and systemic morbidity
B Secondary bloodstream infection
C Death
D A reservoir of drug-resistant microorganisms, and increased health care costs
E All of the above

12. The most common complications of long-term indwelling urinary catheters is:
A Blockage
B Encrustation
C Bacteriuria
D Stones
E Urethral erosions, haematuria and fistula formation

13. The primary pathogen responsible for approximately $80 \%$ of CAUTI is:
A Escherichia coli
B Gram-positive Staphylococci and Enterococci, as well as gram-negative species Proteus
C Klebsiella
D Pseudomonas aeruginosa
E Enterobacter, Candida albicans and other fungal species

14. Which of the following mechanisms entering the urinary tract of bacteria is incorrect?
A The use of silver alloy indwelling catheters for catheterizing
B Upon catheter insertion
C Via ascending colonization that occurs within the catheter lumen (endoluminal) arising from the drainage tubing and collection bag
D Between the catheter and urethra (extraluminal or periurethral)
E Cross-contamination when staff empties the drainage bag 
15. Which of the following statements is incorrect?

A Urinary white blood cells are the best indicator of urinary tract infection.

B Daily bag cleaning with a diluted bleach solution (1:10) is effective in reducing bacterial counts to negligible numbers.

C Silicone catheters and larger lumen size catheters are more resistant to encrustation than other catheter types and smaller lumen size catheters.

D Application of topical antibiotic cream to the meatus around the catheter does reduce bacteriuria.

E The most important intervention to prevent CAUTIs is limiting catheter use.

16. Which of the following statements about general principles of catheter care is incorrect?
A Use a sterile procedure for catheter insertion
B Instilling antibiotics or other additives to the drainage bag
C Use a catheter with the smallest size lumen and balloon possible
D Minimize duration of the catheterization
E Maintain a closed drainage system

17. Which of the following statements about general principles of catheter care is true?
A Antiseptic compounds applied to the meatus
B Keep the collection device below the level of the bladder/tubing
C Specific agents used for meatal cleansing
D Systemic antibiotics for prophylaxis
E Cranberry juice may be helpful in preventing recurring

18. How long can a "Long-Term" Urethral Catheter stay in place? (Before needing to be changed)
A Up to 4 weeks
B Up to 8 weeks
C Up to 12 weeks
D Up to 18 weeks
E Up to 20 weeks

Answers: 1-C, 2-A, 3-E, 4-E, 5-B, 6-B, 7-B, 8-C, 9-D, 10-D, 11-E, 12-C, 13-A, 14-A, 15-D, 16-B, 17-B, 18-C 